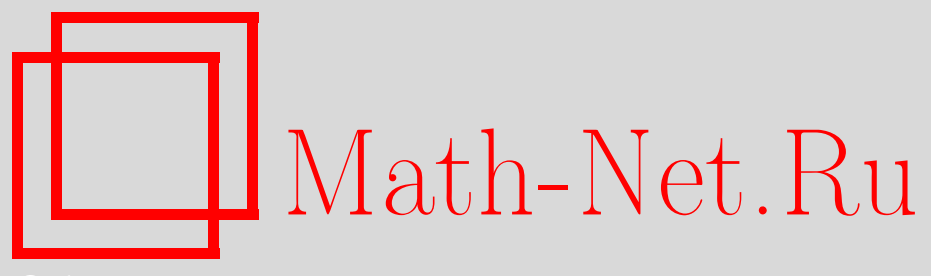

А. И. Штерн, Сильная и слабая непрерывность представлений топологически псевдополных групп в локально выпуклых пространствах, Матем. сб., 2006, том 197, номер 3, 155-176

DOI: https://doi.org/10.4213/sm1540

Использование Общероссийского математического портала Math-Net.Ru подразумевает, что вы прочитали и согласны с пользовательским соглашением http://www . mathnet.ru/rus/agreement

Параметры загрузки:

IP: 3.85 .5 .30

26 апреля 2023 г., 15:06:39

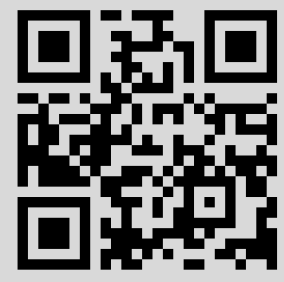




\title{
А. И. Штерн \\ Сильная и слабая непрерывность представлений топологически псевдополных групп в локально выпуклых пространствах
}

\begin{abstract}
Рассматриваются условия слабой и сильной непрерывности представлений топологических групп в локально выпуклых пространствах. В частности, рассматриваются условия слабой непрерывности приводимых локально эквинепрерывных представлений топологической группы в локально выпуклом пространстве, определяющих слабо непрерывные представления в инвариантном подпространстве и в факторпространстве по этому инвариантному подпространству. Эти условия помогают доказать слабую непрерывность усреднений и аппроксимаций, связанных со слабо непрерывными локально эквинепрерывными квазипредставлениями аменабельных топологических групп. Условия сильной непрерывности представления, аппроксимирующего такое квазипредставление, связаны с условиями автоматической сильной непрерывности слабо непрерывных представлений, которые выполняются далеко не для всех групп, пространств и представлений. В связи с этим для широкого класса топологически псевдополных групп (включающего полные по Чеху группы и локально псевдокомпактные группы) указаны условия сильной непрерывности слабо непрерывных представлений в квазиполных бочечных локально выпуклых пространствах. Разобраны некоторые примеры, в частности, связанные с конструкцией $\Sigma$-произведений с отмеченными подгруппами.
\end{abstract}

Библиография: 50 названий.

\section{§ 1. Введение}

Статья посвящена условиям слабой и сильной непрерывности представлений топологических групп в локально выпуклых пространствах и во многом стимулирована замечательной работой Лоуренса Брауна [1], которая, в свою очередь, возникла в связи с работами Клауса Томсена [2], [3] по эквивариантной $K$-теории. Результаты, полученные в работе [1], были достаточны для решения задач, рассматривавшихся в [3], но в работах ряда авторов (в том числе самого Томсена [2], Кунца [4], [5] и Беггса [6]) изучались варианты $K$-теории для случая локально выпуклых алгебр, и в этой теории новые связи между слабой и сильной непрерывностью представлений топологических групп представляют интерес.

Работа выполнена при поддержке Российского фонда фундаментальных исследований (грант № 02-01-00574) и Программы поддержки ведущих научных школ РФ (грант № НШ$619.2003 .1)$. 
В статье получены условия слабой непрерывности приводимых локально эквинепрерывных представлений общих топологических групп в локально выпуклых пространствах, определяющих слабо непрерывные представления в инвариантном подпространстве и в факторпространстве по этому инвариантному подпространству. Эти условия позволяют получить явный вид приводимых слабо непрерывных локально эквинепрерывных квазипредставлений аменабельных топологических групп, определяющих обычные представления в инвариантном подпространстве и в факторпространстве по этому инвариантному подпространству, и установить слабую непрерывность аппроксимирующего представления, поправки и некоторых (инвариантных) средних значений как функций на группе. Условия сильной непрерывности аппроксимирующего представления связаны с условиями автоматической сильной непрерывности слабо непрерывных представлений, которые выполняются не для всех групп и пространств. В статье для широкого класса топологически псевдополных групп (включающего группы полные по Чеху и локально псевдокомпактные группы) указаны условия сильной непрерывности слабо непрерывных представлений в квазиполных бочечных локально выпуклых пространствах, улучшающие соответствующие утверждения статьи [7]. Разобраны некоторые примеры, в частности, связанные с новой конструкцией топологических групп " $\Sigma$-произведением с отмеченными подгруппами".

\section{§ 2. Обозначения и терминология}

Мы пользуемся терминологией и обозначениями теории локально выпуклых пространств, принятыми в книге [8], без дополнительных напоминаний (заметим здесь, что символ * всегда означает двойственное пространство, сопряженный оператор или дуальное представление, а $\sigma(E, F)$ - слабую топологию на локально выпуклом пространстве $E$, индуцированную двойственностью $\{E, F\})$. Терминология, связанная с псевдокомпактными группами, заимствована из работ [9]-[11]. Для информации по теории представлений можно использовать работы [12] и [13], по теории инвариантных средних - [14] и [15], по общей топологии - [16], а по теории нормированных линейных пространств - [17].

Некоторые понятия, используемые в дальнейшем, необходимо напомнить.

ОПРЕДЕЛЕНИЕ 1. Локально выпуклое пространство $E$ называется $n p o c m p a-$ нством, слабая топология которого имеет свойство Крейна ${ }^{1}$ (или, коротко, пространством Крейна в слабой топологии (в связи с одной из теорем в [19])), если слабо замкнутая уравновешенная выпуклая оболочка любого слабо компактного множества в $E$ слабо компактна. Локально выпуклое пространство $E$ называется слабо полным по фон Нейману (поскольку это условие было введено в [20]), если замыкание любого слабо предкомпактного множества в $E$ слабо компактно.

Приведем наиболее общие утверждения, связанные с этими понятиями.

ПреДлОЖенИЕ 1. Слабо квазиполное (т.е. квазиполное в слабой топологии) локалъно выпуклое пространство является слабо полным по фон Нейману, слабо полное по фон Нейману локально выпуклое пространство является

\footnotetext{
${ }^{1}$ Или вафелъно полным ( wafer complete, см. [18; гл. 2]).
} 
пространством Крейна в слабой топологии. Локально выпуклое пространство является пространством Крейна в слабой топологии тогда и только тогда, когда слабо замкнутая выпуклая оболочка любого слабо компактного множества в Е слабо компактна.

ДокАЗАТЕЛЬство. Если локально выпуклое пространство $E$ слабо квазиполно, т.е. любое замкнутое слабо ограниченное подмножество в $E$ слабо полно, то $E$ полно по фон Нейману, поскольку выпуклая оболочка любого слабо предкомпактного множества $B$ в $E$ слабо предкомпактна, и поэтому замыкание выпуклой оболочки множества $B$ ограничено и слабо компактно. В свою очередь, если $E$ полно по фон Нейману, то $E$ является и пространством Крейна в слабой топологии согласно [8; утверждение IV.11.5]. Если выпуклая уравновешенная оболочка любого слабо компактного множества в $E$ слабо компактна, то и выпуклая оболочка любого слабо компактного множества в $E$ слабо компактна. Последнее утверждение предложения также справедливо, поскольку слабо замкнутая уравновешенная выпуклая оболочка любого слабо компактного множества в $E$ является уравновешенной оболочкой слабо замкнутой выпуклой оболочки этого слабо компактного множества (см. [8; I.5.1]).

\section{§ 3. Предварительные замечания}

Как хорошо известно, любое слабо непрерывное линейное представление локально компактной топологической группы в банаховом пространстве сильно непрерывно. Согласно [21], где это замечательное утверждение было впервые опубликовано (с несколько сжатым доказательством), его следует называть теоремой Миркила. Полное и подробное изложение этого утверждения (на языке банаховых модулей над банаховыми алгебрами) приведено в монографии Джонсона [22; с. 23-27]. ${ }^{2}$ Р. Мур [18] распространил этот результат на случай представлений локально компактной группы в бочечном локально выпуклом топологическом векторном пространстве Крейна в слабой топологии, Мегрелишвили [24], [25] - на случай локально эквинепрерывных представлений общих топологических групп в банаховых пространствах со свойством точек непрерывности и в зубчатых локально выпуклых пространствах (см. [26][28]), а Браун [1] - на случай локально эквинепрерывных представлений топологически полных групп в произвольном метризуемом локально выпуклом пространстве. Браун [1] доказал также теорему (формулировка которой приведена ниже) о слабой непрерывности представлений с сильно непрерывным подпредставлением и сильно непрерывным представлением в соответствующем факторпространстве. Эти результаты показывают, что в теоремах об автоматической сильной непрерывности a priori не обязательно сильно непрерывных представлений должно иметь место некоторое взаимодействие условий, налагаемых на топологическую группу, локально выпуклое пространство и собственно представление. Скажем, чтобы ослабить условия непрерывности, нужно усилить условия, налагаемые на группу и пространство.

\footnotetext{
${ }^{2} \mathrm{~K}$ сожалению, доказательство, приведенное в [23], совершенно недостаточно.
} 
Мы приводим вариант теоремы Брауна [1] о слабой непрерывности приводимых представлений топологических групп в локально выпуклых пространствах в предположении, что данное представление определяет слабо непрерывное подпредставление в инвариантном подпространстве и слабо непрерывное представление в соответствующем факторпространстве (см. §4). Эта теорема позволяет, в частности, явным образом описать структуру приводимых слабо непрерывных локально эквинепрерывных квазипредставлений топологической группы, определяющих слабо непрерывные представления в инвариантном подпространстве и в факторпространстве по этому инвариантному подпространству, в предположении, что группа аменабельна как дискретная группа (см. §5). Оба результата повышают интерес к условиям автоматической сильной непрерывности (в топологии Макки) слабо непрерывных представлений топологических групп. В связи с этим в $\S 6$ вводится новый класс топологических групп, так называемые топологически псевдополные группы, содержащий локально псевдокомпактные группы, топологически полные группы и $\Sigma$ произведения локально компактных групп с отмеченными компактными подгруппами, факторпространства по которым метризуемы, а в $§ 7$ доказывается сильная непрерывность слабо непрерывных локально эквинепрерывных представлений топологически псевдополных групп в бочечных локально выпуклых пространствах. Заключительные замечания высказаны в 8 .

\section{§4. Слабая непрерывность приводимых представлений в локально выпуклых пространствах}

Как и в статье [1], в теореме о слабой непрерывности приводимых представлений ${ }^{3}$ в локально выпуклых пространствах мы не налагаем условий на топологическую группу $G$ (пространство которой, конечно, предполагается вполне регулярным). Мы налагаем (не очень ограничительные) условия на пространство представления $E$, в отличие от статьи [1], в которой никаких условий на локально выпуклое пространство представления не налагается. Для простоты мы используем условие локальной эквинепрерывности представления вместо “условия (5)" статьи [1], которое требует, чтобы для любого $g \in G$ и любой непрерывной полунормы $p$ на $E$ существовала окрестность $U$ точки $g$ в $G$ и такая непрерывная полунорма $q$, что

$$
p(\pi(u) \xi) \leqslant q(\xi) \quad \text { для любых } \quad u \in U \quad \text { и любых } \quad \xi \in E
$$

(в [1] отмечено, что локальная эквинепрерывность следует из "условия (5)", если $G$ топологически полна, а $E$ полно и метризуемо (и именно в этих предположениях в [1] доказано, что слабая непрерывность представлений рассматриваемого вида равносильна их сильной непрерывности; см. обсуждение ниже в 6 после введения класса топологически псевдополных групп)). Напомним формулировку теоремы Брауна, ограничиваясь случаем представлений групп (а не полугрупп, которые рассматривались в [1]).

\footnotetext{
${ }^{3}$ Напомним, что представление $\pi$ группы $G$ в локально выпуклом пространстве $E$ называется слабо (сильно) непрерывным, если для любого вектора $x \in E$ отображение $g \mapsto \pi(g) x$, $g \in G$, непрерывно в слабой (сильной) топологии пространства $E$.
} 
ТЕОРЕМА БРАУНА О ПРИВОДИМЫХ ПРЕДСТАВЛЕНИЯХ [1; ЛемМЫ 5,6$]$. Пусть $G$ - топологическая группа, $E$ - локально выпуклое пространство, $F$ - замкнутое векторное подпространство $E, \mathscr{L}(E)$ - алгебра всех непрерывных линейных операторов в $E, \pi$ - такое слабо непрерывное представление группъ $G$ в пространстве $E$, что

$$
\pi(g) F \subset F \quad \text { для любъх } g \in G .
$$

Предположим, что представление $\pi$ удовлетворяет сформулированному выше “условию (5)" и представления группы $G$ в подпространстве $F$ и в факторпространстве $E / F$, определяемые представлением $\pi$, силъно непрерывны. Тогда $\pi$ - слабо непрерывное представление группы $G$ в $E$.

Нетрудно видеть, что некоторые условия приведенной ниже теоремы 1 несколько более ограничительны, чем соответствующие условия теоремы Брауна [1]. С другой стороны, условие сильной непрерывности представлений в подпространстве и в факторпространстве оказалось возможным заменить условием слабой непрерывности, так что результат в теореме 1 выглядит более естественным.

Напомним определение слабой операторной топологии на $\mathscr{L}(E, F)$. Эта топология индуцирована системой полунорм

$$
T \mapsto|f(T x)|, \quad x \in E, \quad f \in F^{*}
$$

(где $F^{*}$ - пространство, двойственное к $F$ ). Если пространство $F$ двойственно к локально выпуклому пространству $F_{*}$, то слабой* операторной топологией на $\mathscr{L}(E, F)$ (связанной с преддвойственным пространством $F_{*}$ ) называется топология, индуцированная системой полунорм

$$
T \mapsto|f(T x)|, \quad x \in E, \quad f \in F_{*} .
$$

Теорема 1. Пусть $G$ - топологическая группа, $E$ - бочечное локально выпуклое пространство, $F$ - замкнутое векторное подпространство в $E, \mathscr{L}(E)$ - алгебра всех непрерывных линейных операторов в $E$, , а $\pi$ - такое слабо непрерывное представление $G$ в $E$, что

$$
\pi(g) F \subset F \quad \text { для любъх } g \in G .
$$

Предположим, что $\pi$ локально слабо ограничено (т.е. образ $\pi(U)$ некоторой окрестности $U$ единицы е в $G$ слабо ограничен в $\mathscr{L}(E))$ и что представления группы $G$ в подпространстве $F$ и в факторпространстве $E / F$, определяемые представлением $\pi$, слабо непрерывны. Тогда $\pi$ - слабо непрерывное представление группы $G$ в $E$.

ДокАЗАтЕльство. Пусть $\pi^{*}$ - представление группы $G$ в $E^{*}$, определенное формулой

$$
\pi^{*}(g)=\left(\pi\left(g^{-1}\right)\right)^{*}, \quad g \in G,
$$

где звездочка в правой части равенства означает переход к сопряженному оператору. Достаточно доказать, что $\pi^{*}$ - слабо* непрерывное представление группы $G$ в $E$ (здесь и далее под слабой* операторной топологией мы понимаем топологию, связанную с пространством $E$, преддвойственным к $E^{*}$ ). 
Пусть $U$ - такая симметричная окрестность единицы $e$ в $G$, что множество $H=\pi(U)$ ограничено в топологии простой сходимости, связанной со слабой топологией в $E$ (т.е. с $\sigma\left(E, E^{*}\right)$-топологией). Поэтому множество $H^{*}=\pi^{*}(U)$ ограничено в слабой* операторной топологии на пространстве $\mathscr{L}\left(E^{*}\right)$.

По предположению пространство $E$ бочечно. Поэтому из принципа равномерной ограниченности [8; теорема III.4.2] следует, что множество $H^{*} f$ эквинепрерывно в $E^{*}$ для любого $f \in E^{*}$. Поскольку пространство, сопряженное к бочечному, квазиполно в слабой* топологии (согласно III.4.4 и очевидному слабому* варианту утверждения III.4.3 в [8]), то замыкание $\bar{H}^{*}$ множества $H^{*}$ в $\mathscr{L}\left(E^{*}\right)$ компактно в слабой* операторной топологии (доказательство использует стандартные рассуждения, связанные с компактностью произведения компактных множеств $\bar{H}^{*} f$ в $E^{*}$ для любых $\left.f \in E^{*}\right)$.

Воспользуемся приемом, работавшим в [7] и [29] в аналогичных ситуациях. Пусть $M$ - пересечение замыканий $\overline{\pi^{*}(V)}$ (здесь и далее черта над подмножеством пространства $\mathscr{L}\left(E^{*}\right)$ означает замыкание этого подмножества в указанной выше слабой* операторной топологии $\left.{ }^{4}\right)$ образов $\pi^{*}(V)$ всевозможных симметричных окрестностей $V \subset G$ единицы в $G$. Так как мы доказали, что замыкание множества $\pi^{*}(U)$ компактно, то множество $\overline{\pi^{*}(V)}$ компактно в слабой* операторной топологии для любых симметричных окрестностей $V \subset U$ (поскольку множество $\overline{\pi^{*}(V)}$ замкнуто в компактном множестве).

Ввиду упомянутой выше компактности множества $\overline{\pi^{*}(V)}$ соотношение $M=$ $\left\{1_{E^{*}}\right\}$ эквивалентно слабой* непрерывности представления $\pi^{*}$ в единице $e$. Действительно, пополнение любой окрестности $W$ нулевого элемента в множестве $\overline{\pi^{*}\left(V_{0}\right)}$ (для любой такой окрестности $V_{0}$, что $\overline{\pi^{*}\left(V_{0}\right)}$ компактно в слабой* операторной топологии) тоже компактно в слабой* операторной топологии. Если $M=\left\{1_{E^{*}}\right\}$, то пересечение $\left.M \cap \overline{\left(\pi^{*}\left(V_{0}\right)\right.} \backslash W\right)$ пусто и поэтому существует конечное семейство множеств вида $\overline{\pi^{*}\left(V_{i}\right)}, i=1, \ldots, N$, для которых пересечение

$$
\left(\bigcap_{i=1}^{N} \overline{\pi^{*}\left(V_{i}\right)}\right) \cap\left(\overline{\pi^{*}\left(V_{0}\right)} \backslash W\right)
$$

пусто, так что соотношение $\pi^{*}(V) \subset W$ выполняется для любой окрестности

$$
V=V_{0} \cap\left(\bigcap_{i=1}^{N} V_{i}\right) .
$$

Пусть $T$ - элемент $M$. Докажем, что $T=1_{E^{*}}$. Если $f \in F^{\perp}$, где $F^{\perp}-$ аннулятор пространства $F$ в $E^{*}$, то $T f=f$. Поскольку представление, определенное представлением $\pi$ в $E / F$, слабо непрерывно, то ограничение представления $\pi^{*}$ на $F^{\perp}$ слабо* непрерывно. Из указанной выше эквивалентности соотношения вида $M=\left\{1_{E^{*}}\right\}$ и условия слабой* непрерывности соответствующего представления в единице $е$ группы $G$ следует, что часть оператора $T$, отвечающая подпространству $F^{\perp}$, совпадает с единичным оператором на $F^{\perp}$. Аналогично, представление, определенное представлением $\pi^{*}$ в $E^{*} / F^{\perp}$, тоже

\footnotetext{
${ }^{4}$ Из [8; теорема III.4.4 и упражнение 25 к гл. IV] следует, что операторы вида $T$ непрерывны как операторы в сопряженном пространстве, снабженном слабой топологией.
} 
слабо* непрерывно и соответствующий фактороператор, определенный оператором $T$, совпадает с единичным оператором на $E^{*} / F^{\perp}$. Таким образом, если $f \in E^{*}$, то $T f-f \in F^{\perp}$.

Пусть $T \neq 1_{E^{*}}$. Тогда существует такой функционал $f_{0} \in E^{*}$, что $T f_{0} \neq f_{0}$. Мы уже доказали, что элемент $g=T f-f$ принадлежит $F^{\perp}$, причем $g \neq 0$. Итак, $T f=f+g$. Тогда

$$
T^{2} f=T(T f)=T f+T g=(f+g)+g=f+2 g
$$

и по индукции получаем, что

$$
T^{n} f=f+n g \quad \text { для любых } n \in \mathbb{N} .
$$

С другой стороны, для любой окрестности $V$ единицы в $G$ и любого натурального $n$ существует такая окрестность $W$ единицы в $G$, что $W^{2^{n}} \subset V$. Поэтому

$$
\pi^{*}(W)^{2^{n}} \subset \pi^{*}(V) .
$$

Отсюда следует, что

$$
\left(\pi^{*}(W)\right)^{2^{n}} \subset \pi^{*}(V) \subset \overline{\pi^{*}(V)} .
$$

Пусть $n=1$. Тогда

$$
\pi^{*}(W) \pi^{*}(W) \subset \pi^{*}(V) .
$$

Для любого $B \in \pi^{*}(W)$ из очевидного соотношения

$$
B \pi^{*}(W) \subset \pi^{*}(V)
$$

следует, что

$$
B \overline{\pi^{*}(W)} \subset \overline{\pi^{*}(V)}
$$

и поэтому

$$
\pi^{*}(W) \overline{\pi^{*}(W)} \subset \overline{\pi^{*}(V)} .
$$

В свою очередь, для любого $B \in \overline{\pi^{*}(W)}$ из очевидного соотношения

$$
\pi^{*}(W) B \subset \overline{\pi^{*}(V)}
$$

следует, что

$$
\overline{\pi^{*}(W)} B \subset \overline{\pi^{*}(V)}
$$

и поэтому

$$
\overline{\pi^{*}(W)} \overline{\pi^{*}(W)} \subset \overline{\pi^{*}(V)} .
$$

По индукции отсюда следует, что для любой окрестности $V$ единичного элемента в $G$, любого натурального $n$ и любой такой окрестности $W$ единицы в $G$, что $W^{2^{n}} \subset V$, имеем

$$
\left(\overline{\pi^{*}(W)}\right)^{2^{n}} \subset \overline{\pi^{*}(V)} .
$$

В частности, поскольку $T \in \overline{\pi^{*}(O)}$ для любой окрестности $O$ единицы, отсюда следует, что

$$
T^{n} \in \overline{\pi^{*}(V)} \quad \text { для любого } n \in \mathbb{N}
$$

и любой окрестности $V$ единицы в $G$ и поэтому $T^{n} \in M$. Однако, как мы видели выше, если $T \neq 1_{E^{*}}$, то последовательность $n \mapsto T^{n}, n \in \mathbb{N}$, не ограничена, а это противоречит условию, что образ $\pi^{*}(U) \subset \mathscr{L}(E)$ некоторой окрестности $U$ элемента $е$ ограничен. Отсюда следует, что $T=1_{E}$, что завершает доказательство теоремы 1 . 


\section{§5. Аппроксимация приводимого слабо непрерывного локально эквинепрерывного квазипредставления аменабельной топологической группы в банаховом пространстве}

Полученные в 22 условия слабой непрерывности позволяют установить существование и слабую непрерывность аппроксимирующего представления для любого приводимого слабо непрерывного локально эквинепрерывного квазипредставления топологической группы, для которого квазипредставления, определяемые данным квазипредставлением в инвариантном подпространстве и в факторпространстве по этому инвариантному подпространству, являются обычными представлениями. Определения и обозначения см., например, в работах [29] и [30]. Напомним, в частности, что отображение $\theta$ группы $G$ в группу обратимых элементов алгебры $\mathscr{L}(E)$ ограниченных линейных операторов в $E$ называется $\delta$-квазипредставлением группы $G$ в $E$, если $\|\theta(g h)-\theta(g) \theta(h)\| \leqslant \delta$ для всех $g, h \in G$.

Теорема 1.3 в [30] допускает следующий специфический вариант, связанный с рассматриваемой задачей.

Теорема 2. Пусть $G$ - аменабельная топологическая группа, $E$ - сопряженное банахово пространство, $F$ - замкнутое векторное подпространство в $E$, , а $\theta$ - такое слабо непрерывное $\delta$-квазипредставление топологической группь $G$ в $E$, что

$$
\theta(g) F \subset F \quad \text { для любъх } g \in G .
$$

Предположим, что $\theta$ локально равномерно ограничено, $\theta(e)=1_{E} u \theta\left(g^{-1}\right)=$ $\theta(g)^{-1}$ для всех $g \in G$. Предположим также, что и ограничение представления $\theta$ на $F$, и отображение, определяемое отображением $\theta$ в $E / F$, являются обычными представлениями. Тогда существует такое слабо непрерывное представление $\pi$ группь $G$ в $E$, что $\|\pi(g)-\theta(g)\| \leqslant \delta$ для всех $g \in G$ и представления, определяемые представлением $\pi$ в $F$ u $E / F$, совпадают с представлениями, определяемыми квазипредставлением $E$ в $F$ и $E / F$ соответственно.

ДокАЗАТЕЛЬство. Так как $\theta-\delta$-квазипредставление, для всех $g, h \in G$ имеем

$$
\left\|\theta(g h) \theta\left(h^{-1}\right)-\theta(g)\right\| \leqslant \delta .
$$

В частности, $h \mapsto \theta(g h) \theta\left(h^{-1}\right)$ - ограниченная операторнозначная функция со значениями в $\mathscr{L}(E)$. Пусть $E_{*}-$ (некоторое) банахово пространство, к которому сопряжено пространство $E$. Положим

$$
\pi(g)=I_{h}\left(\theta(g h) \theta\left(h^{-1}\right)\right) \text { для любого } g \in G,
$$

где $I_{h}(F(h))$ для ограниченной операторной функции $F$ на $G$ со значениями в пространстве ограниченных операторов в $E$ понимается как оператор, сопоставляющий элементу $x \in E$ элемент $y \in E$, определенный формулой

$$
y(f)=I_{h \in G}((F(g) x)(f)) \quad \text { для любого } \quad f \in E_{*}
$$


для некоторого фиксированного двусторонне инвариантного среднего $I$ на пространстве ограниченных непрерывных функций переменного $h \in G$ на группе $G$. По построению оператор $\pi(g)-\theta(g)$ удовлетворяет условию

$$
\|\pi(g)-\theta(g)\| \leqslant \delta
$$

которое сразу следует из неравенства (1), а из соотношения

$$
\pi(g)-\theta(g)=I_{h}\left((\theta(g h)-\theta(g) \theta(h)) \theta\left(h^{-1}\right)\right)
$$

следует, что оператор $\pi(g)-\theta(g)$ действует из $E$ в $F \subset E$ и равен нулю на $F$. Напомним также, что все произведения вида

$$
(\theta(g h)-\theta(g) \theta(h))(\theta(k l)-\theta(k) \theta(l)), \quad g, h, k, l \in G,
$$

равны нулю на $E$. Ввиду двусторонней инвариантности среднего $I$ имеем

$$
\begin{gathered}
\pi(g)=I_{h}\left(\theta(g h) \theta\left(h^{-1}\right)\right)=I_{h}\left(\theta(g k h) \theta\left((k h)^{-1}\right)\right)=I_{h}\left(\theta(g k h) \theta\left(h^{-1} k^{-1}\right)\right. \\
\left.\quad+\theta(g k) \theta(h)\left(\theta\left(h^{-1} k^{-1}\right)-\theta\left(h^{-1}\right) \theta\left(k^{-1}\right)\right)+\theta(g k h) \theta\left(h^{-1}\right) \theta\left(k^{-1}\right)\right) \\
=\theta(g k) I_{h}\left(\theta(h)\left(\theta\left(h^{-1} k^{-1}\right)-\theta\left(h^{-1}\right) \theta\left(k^{-1}\right)\right)\right)+\pi(g k) \theta\left(k^{-1}\right)
\end{gathered}
$$

для всех $g, k \in G$. Из соотношения $\theta(h)^{-1}=\theta\left(h^{-1}\right)$, где $h \in G$, следует, что

$$
I_{h}\left(\theta(h)\left(\theta\left(h^{-1} k^{-1}\right)-\theta\left(h^{-1}\right) \theta\left(k^{-1}\right)\right)\right)=I_{h}\left(\theta(h) \theta\left(h^{-1} k^{-1}\right)\right)-\theta\left(k^{-1}\right),
$$

а из инвариантности среднего (с помощью замены $h \longmapsto k l$ и перехода к среднему по $l$ ) заключаем, что

$$
I_{h}\left(\theta(h) \theta\left(h^{-1} k^{-1}\right)\right)-\theta\left(k^{-1}\right)=I_{l}\left(\theta\left(k^{-1} l\right) \theta(l)\right)-\theta\left(k^{-1}\right)=\pi\left(k^{-1}\right)-\theta\left(k^{-1}\right) .
$$

Таким образом,

$$
\pi(g)=\theta(g k)\left(\pi\left(k^{-1}\right)-\theta\left(k^{-1}\right)\right)+\pi(g k) \theta\left(k^{-1}\right)
$$

или

$$
\theta(g k) \theta\left(k^{-1}\right)-\theta(g k) \pi\left(k^{-1}\right)-\pi(g k) \theta\left(k^{-1}\right)+\pi(g)=0_{E}
$$

для всех $g, k \in G$. С другой стороны, имеем

$$
\begin{aligned}
0_{E} & =(\pi(g k)-\theta(g k))\left(\pi\left(k^{-1}\right)-\theta\left(k^{-1}\right)\right) \\
& =\pi(g k) \pi\left(k^{-1}\right)-\theta(g k) \pi\left(k^{-1}\right)-\pi(g k) \theta\left(k^{-1}\right)+\theta(g k) \theta\left(k^{-1}\right)
\end{aligned}
$$

для всех $g, k \in G$ и можно вычесть последнее тождество из предыдущего. Получаем, что

$$
\pi(g)-\pi(g k) \pi\left(k^{-1}\right)=-(\pi(g k)-\theta(g k))\left(\pi\left(k^{-1}\right)-\theta\left(k^{-1}\right)\right)=0_{E},
$$

т.е. $\pi$ - представление, причем $\pi(g)-\theta(g)$ есть оператор из $E$ в $F$. Таким образом, представления, определяемые представлением $\pi$ в $F$ и $E / F$, совпадают с представлениями, определяемыми квазипредставлением $E$ в $F$ и $E / F$ соответственно. Ввиду локальной равномерной ограниченности представления $\pi$ и слабой непрерывности представлений, определяемых квазипредставлением $E$ в $F$ и $E / F$, представление $\pi$ слабо непрерывно по теореме 1. 
Отсюда немедленно получаем

СледСтвиЕ 1. Пусть $G$ - аменабельная топологическая группа, $E$ - сопряженное банахово пространство, $F$ - замкнутое векторное подпространство в $E$, а $\theta$ - такое слабо непрерывное $\delta$-квазипредставление топологической группь $G$ в $E$, что

$$
\theta(g) F \subset F \quad \text { для любъх } g \in G .
$$

Предположим, что $\theta(e)=1_{E}, \theta$ локально равномерно ограничено и соотношения $\theta\left(g^{-1}\right)=\theta(g)^{-1}$ выполнены для всех $g \in G$. Предположим также, что ограничение представления $\theta$ на $F$ и отображение, определяемое отображением $\theta$ в $E / F$, являются обычными представлениями. Тогда функиия

$$
g \mapsto I_{h}\left(\theta(g h) \theta\left(h^{-1}\right)\right), \quad g \in G,
$$

слабо непрерывна как функция со значениями в непрерывных линейных операmopax в $E$.

ЗАмечАниЕ. Напомним, что среднее от непрерывной функции двух переменных по одной из переменных не обязательно непрерывно. Например, среднее от функции

$$
\{x, y\} \mapsto \exp (i x y), \quad x, y \in \mathbb{R},
$$

по переменной $x$ определено однозначно и разрывно как функция от $y$.

СлЕДСТвиЕ 2. Пусть выполнены условия теоремы 2, и пусть $\pi$ - представление, существование которого утверждается в теореме 2, а $\delta: G \rightarrow$ $\mathscr{L}(E)$ - ограниченное отображение $(\|\delta(g)\| \leqslant f(\varepsilon))$, являющееся поправкой (т.е. $\pi=\theta+\delta)$. Если семейство операторов $\left\{\theta(g) \pi\left(g^{-1}\right) \mid g \in G\right\}$ (или, что равносильно, семейство операторов $\left.\left\{\pi(g) \theta\left(g^{-1}\right) \mid g \in G\right\}\right)$ ограничено, то отображение

$$
h \mapsto I_{h}\left(\delta(g h) \theta\left(h^{-1}\right)\right), \quad g, h \in G,
$$

определено и слабо непрерывно по $h \in G$ как отображение группъ $G$ в алгебру $\mathscr{L}(E)$.

ДокАЗАТЕЛЬство. Пусть $\pi$ - представление, построенное в теореме 2 , и пусть $\delta: G \rightarrow \mathscr{L}(E)$ - ограниченное отображение $(\|\delta(g)\| \leqslant f(\varepsilon))$, являющееся поправкой (т.е. $\pi=\theta+\delta$ ). Так как

$$
\pi(g h)-\pi(g) \pi(h) \equiv 0_{E} \quad \text { для всех } \quad g, h \in G,
$$

TO

$$
\theta(g h)+\delta(g h)-\theta(g) \theta(h)-\delta(g) \theta(h)-\theta(g) \delta(h)-\delta(g) \delta(h)=0_{E}
$$

для любых $g, h \in G$. Очевидно, $\delta(g) \delta(h) \equiv 0_{E}$. Следовательно,

$$
\delta(g)=\theta(g h)(\theta(h))^{-1}-\theta(g)-\theta(g) \delta(h)(\theta(h))^{-1}+\delta(g h)(\theta(h))^{-1}
$$

для любых $g, h \in G$ и поэтому правая часть равенства не зависит от $h \in G$. Кроме того, отображение

$$
\{g, h\} \mapsto \theta(g h)(\theta(h))^{-1}-\theta(g)=\theta(g h) \theta\left(h^{-1}\right)-\theta\left(g h h^{-1}\right), \quad g, h \in G,
$$


ограничено. Применяя слабое* инвариантное среднее по $h \in G$, как в [30], видим, что

$\pi(g)-\theta(g)=\delta(g)=\pi(g)-\theta(g)+\delta(g) w+I_{h}\left(\delta(g h) \theta\left(h^{-1}\right)\right) \quad$ для любого $g \in G$,

где $w$ - инвариантное среднее в слабой операторной топологии от функции $\delta(h) \theta\left(h^{-1}\right)$, взятое по переменной $h \in G$ (эта функция ограничена, если ограничено семейство операторов $\left\{\theta(g) \pi\left(g^{-1}\right) \mid g \in G\right\}$ или, что равносильно, если ограничено семейство операторов $\left.\left\{\pi(g) \theta\left(g^{-1}\right) \mid g \in G\right\}\right)$. Таким образом,

$$
I_{h}\left(\delta(g h) \theta\left(h^{-1}\right)\right)=-\delta(g) w \quad \text { для любого } g \in G,
$$

что и доказывает слабую непрерывность отображения

$$
g \mapsto I_{h}\left(\delta(g h) \theta\left(h^{-1}\right)\right), \quad g, h \in G .
$$

\section{§ 6. Топологически псевдополные группы}

В 44 были получены условия слабой непрерывности представлений топологических групп в бочечных локально выпуклых пространствах, а в предыдущем параграфе эти условия непрерывности позволили нам установить слабую непрерывность представлений, аппроксимирующих слабо непрерывные локально эквинепрерывные квазипредставления топологической группы, определяющие обычные представления в инвариантном подпространстве и в факторпространстве по этому инвариантному подпространству. Естественно возникает вопрос об общих условиях сильной непрерывности представлений этого класca.

Нетрудно видеть, что условия сильной непрерывности аппроксимирующего представления связаны с условиями автоматической сильной непрерывности слабо непрерывных представлений, которые выполняются далеко не для всех групп, пространств и представлений.

Пример. Пусть $E$ - бесконечномерное пространство, пусть $F \neq E$ - бесконечномерное замкнутое векторное подпространство в $E$ с бесконечномерным факторпространством $E / F$, и пусть $G$ - группа всех непрерывных линейных операторов в $E$, отличающихся от единичного оператора на конечномерный линейный оператор вида

$$
T x=\sum_{i=1}^{n} f_{i}(x) x_{i}, \quad x \in E, \quad x_{i} \in F, \quad f_{i} \in F^{\perp}, \quad n \in \mathbb{N},
$$

где $F^{\perp}$ - аннулятор пространства $F$ в $E^{*}$. Снабдим группу $G$ слабой операторной топологией. Нетрудно видеть, что $G$ - топологическая группа и тавтологическое представление группы $G$ в $E$, очевидно, слабо непрерывно, но не сильно непрерывно, так как в бесконечномерном банаховом пространстве слабая и сильная топологии различны.

В этом параграфе мы напомним определение класса топологических групп, введенного в работе [7]. Начнем с определения псевдокомпактных и локально псевдокомпактных групп. 
ОПРЕДЕЛЕНИЕ 2. Топологическое пространство называется псевдокомпакmным, если любая вещественнозначная непрерывная функция на этом пространстве ограничена. Топологическое пространство $X$ называется локально псевдокомпактным, если любая точка пространства $X$ имеет псевдокомпактную окрестность. Топологическая группа называется псевдокомпактной (локально псевдокомпактной), если топологическое пространство этой группы псевдокомпактно (локально псевдокомпактно).

Нетрудно видеть, что метризуемые псевдокомпактные группы компактны. Дополнительную информацию о псевдокомпактных группах, локально псевдокомпактных группах и их обобщениях можно найти в работах [9]-[11], [31]-[33].

Введем класс групп (содержащий, в частности, класс локально псевдокомпактных групп), слабо непрерывные представления которых в "хороших" локально выпуклых пространствах автоматически сильно непрерывны, см. теорему 3 ниже. Напомним, что топологическая группа $G$ называется топологически полной или почти метризуемой [34], [35], если $G$ допускает компактификацию, в которой исходная группа $G$ образует множество типа $G_{\delta}$ или, что равносильно, если топологическая группа $G$ содержит компактную подгруппу $N$ с метризуемым пространством смежных классов $G / N$. Мы расширим этот класс следующим образом.

ОПРЕДЕЛЕНИЕ 3. Топологическая группа $G$ называется топологически nceвдополной, если она содержит замкнутую псевдокомпактную подгруппу $N$ с метризуемым пространством смежных классов $G / N$.

Очевидно, что группа $N$ есть множество типа $G_{\delta}$ в $G$.

Как показывает следующее утверждение, класс топологически псевдополных групп довольно широк.

ПРЕДЛОЖЕНИЕ 2. Все топологически полные топологические группы и все локально псевдокомпактные топологические группы принадлежат классу топологически псевдополных групп.

В доказательстве этого предложения нам потребуется понятие пополнения Вейля псевдокомпактной группы. Напомним, что любая псевдокомпактная топологическая группа $G$ может быть так вложена в виде плотной подгруппы в некоторую локально компактную группу $K$, что любая непрерывная (автоматически ограниченная) вещественная функция на $G$ допускает (и притом единственное) непрерывное (автоматически ограниченное) продолжение до вещественной функции на $K$. (Пространство группы $K$ совпадает тем самым со стоун-чеховской компактификацией группы $G$.) Это условие определяет топологическую группу $K$ однозначно с точностью до взаимно непрерывного изоморфизма групп, поточечно сохраняющего группу $G$. Группа $K$ называется пополнением Вейля группы $G$. Подробности можно найти в работах [9]-[11], [31]-[33].

ДокАзАтельство. Первое утверждение предложения 2 очевидно. Второе утверждение вытекает из одного из результатов статьи [10]: топологическая группа $G$ локально псевдокомпактна тогда и только тогда, когда пространство смежных классов (однородное пространство) $G / N$ локально компактно для любой замкнутой $G_{\delta}$-подгруппы $N$ в $G$. Действительно, если $G$ локально 
псевдокомпактна и $K$ - пополнение Вейля группы $G$ и если $H$ - открытая почти связная подгруппа в $K$, то подгруппа $G^{\prime}=G \cap H$ в $G$ очевидным образом локально псевдокомпактна, так как достаточно малые окрестности любой точки группы $G^{\prime}$ в группе $G^{\prime}$ и в $G$ одни и те же. Любая окрестность $U$ единицы в $H$ содержит такой замкнутый нормальный делитель $M_{U}$, что соответствующая факторгруппа $H / M_{U}$ является конечным расширением связной группы Ли и, тем самым, нормальный делитель $M_{U}$ есть $G_{\delta}$-множество в $H$. Рассмотрим соответствующий непрерывный канонический гомоморфизм $H \rightarrow H / M_{U}$ и ограничим его на $G^{\prime}$. Мы получим непрерывный гомоморфизм $G^{\prime} \rightarrow H / M_{U}$, ядро которого принадлежит данной окрестности в $H$ (или, что равносильно, данной окрестности в $G$ ) и имеет метризуемый образ (или, что равносильно, метризуемую факторгруппу). Так как $G^{\prime}$ открыта в $G$, то факторпространство $G / N$ также метризуемо (см. [16; гл. $\mathrm{V}, \S 5.5]$ и $[36 ; \S 8]$ ), что завершает доказательство.

Введем следующую групповую конструкцию (которая, по-видимому, является новой).

ОПРЕДЕЛЕНИЕ 4. Пусть $G_{\alpha}, \alpha \in A$, - семейство топологических групп, и пусть $H_{\alpha}$ - открытая компактная подгруппа группы $G_{\alpha}$ для любого $\alpha \in A$. Пусть $G$ - подгруппа прямого произведения $\prod_{\alpha \in A} G_{\alpha}$, образованная всеми такими элементами $\left\{g_{\alpha}\right\} \in \prod_{\alpha \in A} G_{\alpha}$, что $g_{\alpha} \neq e_{\alpha}$ лишь для счетного множества индексов $\alpha \in A$, а $g_{\alpha} \notin H_{\alpha}$ лишь для конечного множества индексов $\alpha \in A$. Очевидно, что $G$ - топологическая группа с открытой псевдокомпактной подгруппой $H$, являющейся обычным $\Sigma$-произведением компактных подгрупп $H_{\alpha}, \alpha \in A$. Группа $G$ называется $\Sigma$-произведением топологических групп $G_{\alpha}, \alpha \in A$, с отмеченными подгруппами $H_{\alpha}, \alpha \in A$. Топология в группе $G$ определяется семейством окрестностей, определяемых как пересечение окрестностей в $\prod_{\alpha \in A} H_{\alpha}$ с группой $G$.

Следующее предложение показывает, что класс топологически псевдополных групп содержит $\Sigma$-произведения топологически полных групп $G_{\alpha}, \alpha \in A$, с отмеченными открытыми компактными подгруппами.

ПрЕДЛОЖЕНИЕ 3. Если $G_{\alpha}, \alpha \in A$, - семейство топологически полных групп с открытыми компактными открытыми подгруппами $H_{\alpha}$, то $\Sigma$-произведение групп $G_{\alpha}, \alpha \in A$, с отмеченными подгруппами $H_{\alpha}$ является топологически псевдополной группой.

ДокАЗАТЕЛЬство. Группа $G$ - топологическая группа с псевдокомпактной подгруппой $H$, а факторпространство $G / H$ дискретно и потому метризуемо.

Конструкция $\Sigma$-произведения топологических групп с отмеченными подгруппами допускает модификацию, в которой отмеченные подгруппы не обязательно открыты.

Отметим в заключение параграфа, что класс топологически псевдополных групп не исчерпывается объединением классов локально псевдокомпактных и топологически полных групп. Очевидно, что подходящее прямое произведение локально псевдокомпактной группы и топологически полной группы может оказаться группой, которая не локально псевдокомпактна и не топологически полна. 


\section{§ 7. Сильная непрерывность слабо непрерывных представлений топологически псевдополных групп в бочечных локально выпуклых пространствах}

В этом параграфе мы докажем следующее утверждение.

ТеОрема 3. Пусть $G$ - топологически псевдополная топологическая груп$n a, E$ - квазиполное бочечное локально выпуклое пространство, $\mathscr{L}(E)$ - алгебра всех непрерывных линейных операторов в $E, \pi$ - слабо непрерывное представление группы $G$ в пространстве $E$, и пусть $\pi$ локально слабо ограничено (т.е. образ $\pi(U)$ некоторой окрестности $U$ единиць е в $G$ есть слабо ограниченное подмножество алгебры $\mathscr{L}(E))$. Тогда $\pi$ - сильно непрерывное представление группы $G$ в $E$. Если группа $G$ топологически полна, то утверждение теоремы выполняется и в том случае, когда E- пространство Крейна в слабой топологии (не обязательно квазиполное).

Аналогичное утверждение, доказанное в [7], предполагало полноту пространства по фон Нейману, а не квазиполноту в сильной топологии. Нетрудно видеть, что банахово пространство полно по фон Нейману тогда и только тогда, когда оно рефлексивно. Таким образом, утверждение, доказанное в [7], не охватывало случай представлений в нерефлексивных банаховых пространствах. Теорема 3 существенно сильнее теоремы из [7] и свободна от этого недостатка. Таким образом, в частности, локально слабо ограниченные слабо непрерывные представления топологически псевдополных групп в банаховых пространствах сильно непрерывны.

Для доказательства теоремы 3 потребуются некоторые вспомогательные утверждения.

ПРЕДЛОЖЕНИЕ 4. Пусть $G$ - псевдокомпактная топологическая группа, $K$ - пополнение Вейля группы $G, E$ - квазиполное бочечное локально выпуклое пространство, а $\pi$ - слабо непрерывное представление группы $G$ в $E$. Тогда представление $\pi$ сильно непрерывно, а $\pi$ можно продолжить до сильно непрерывного представления компактной группь $K$ в том же пространстве $E$. Если сама группа $G$ компактна, то утверждение теоремы остается справедливым, если пространство Е является всего лишь пространством Крейна в слабой топологии (и не обязательно квазиполным).

Напомним, что псевдокомпактность в бочечных пространствах была связана в работе [37] с относительной слабой компактностью. А именно в [37] доказано, что любое относительно слабо псевдокомпактное множество в квазиполном бочечном локально выпуклом пространстве $E$ (т.е. такое множество $X$, что ограничение любой слабо непрерывной функции на $E$ на множество $X$ ограничено на $X$ ) является относительно слабо компактным в $E .^{5}$

ДокАзАТЕЛьство. Достаточно доказать, что представление $\pi$ можно продолжить до слабо непрерывного представления компактной группы $K$ в том же

\footnotetext{
5 Доказательство в [37] использует понятия и средства, развитые в доказательстве теоремы IV.11.2 в [8] и основано на критерии Гротендика слабой компактности [38]. Из сравнения этих средств следует, что в приведенном утверждении об относительной слабой компактности слабо псевдокомпактных множеств можно несколько ослабить условие квазиполноты. А именно достаточно требовать, чтобы пространство было бочечным и замыкания относительно слабо псевдокомпактных множеств в этом пространстве были полны.
} 
пространстве $E$. Действительно, слабо непрерывное представление любой компактной топологической группы в любом бочечном пространстве, являющемся пространством Крейна в слабой топологии, сильно непрерывно [18]. Таким образом, если требуемое продолжение до представления группы $K$ в $E$ существует, то оно сильно непрерывно. Это рассуждение завершает доказательство теоремы, если группа $G$ компактна.

Построим требуемое расширение в общем случае. По определению псевдокомпактного пространства любая непрерывная вещественная функция на $G$ ограничена. Из определения сразу следует, что непрерывный образ псевдокомпактного топологического пространства псевдокомпактен. Так как представление $\pi$ слабо непрерывно по предположению, то отображение $g \mapsto \pi(g) x, g \in G$, непрерывно как отображение группы $G$ в пространство $E$, снабженное слабой топологией, для любого $x \in E$. Поэтому орбита $O(x)=\{\pi(g) x \mid g \in G\}$ каждого вектора $x \in E$ под действием группы $G$ псевдокомпактна в слабой топологии, т.е. любая слабо непрерывная вещественная функция на $O(x)$ ограничена. Тогда и ограничение любой слабо непрерывной функции на $E$ на множество $O(x)$ ограничено на $O(x)$. Поскольку пространство $E$ квазиполно по предположению, то каждая орбита $O(x)$ слабо относительно компактна по теореме 3 из [37]. Следовательно, замкнутая выпуклая оболочка $H(x)$ этой орбиты слабо предкомпактна (см. [8; I.5.1]) и потому слабо ограничена. Множество $H(x)$ выпукло и замкнуто, а поэтому и слабо замкнуто. Таким образом, множество $H(x)$ слабо компактно для любого $x \in E$. Стандартное рассуждение, использующее теорему Тихонова, показывает тогда, что множество $\pi(G)$ имеет компактное замыкание в слабой операторной топологии пространства $\mathscr{L}(E)$.

Отметим теперь, что любая комплекснозначная непрерывная функция на $G$ тоже ограничена. Кроме того, поскольку компактная группа $K$ является стоунчеховской компактификацией группы $G$, то любая комплекснозначная непрерывная функция на $G$ допускает единственное продолжение по непрерывности до комплекснозначной непрерывной функции на $K$. Так как пространство $E$ бочечно по предположению, то множество $\pi(G)=\{\pi(g) \mid g \in G\}$ эквинепрерывно в пространстве $\mathscr{L}(E)$ по теореме IV.5.2 в [8]. Таким образом, любая точка $k \in K$ определяет ограниченный фильтр на множестве $\pi(G)$, образованный образами пересечений окрестностей точки $k$ в $K$ с множеством $G$. Это - фильтр Коши в слабой операторной топологии, сходящийся к некоторому отображению $\pi(k)$ (ввиду поточечной компактности образов) в пространстве $E^{E}$ всех отображений $E$ в $E$ с топологией простой слабой сходимости. Еще раз используя тот факт, что пространство $E$ бочечно, и применяя теорему III.4.6 из [8], получаем, что $\pi(k) \in \mathscr{L}(E)$ и построенный фильтр сходится к $\pi(k)$ в слабой операторной топологии на пространстве $\mathscr{L}(E)$. Это правило определяет некоторое отображение $K \rightarrow \mathscr{L}(E)$, которое непрерывно в слабой операторной топологии, потому что матричные элементы построенного отображения являются непрерывными функциями на $K$. Остается доказать, что построенное отображение (которое мы обозначаем той же буквой $\pi$, поскольку продолженное отображение однозначно определено исходным представлением $\pi$ ) есть представление группы $K$ в пространстве $E$. Заметим, что

$$
\pi(g h)=\pi(g) \pi(h) \quad \text { для любых } \quad g, h \in G
$$


и поэтому

$$
f(\pi(g h) \xi)=f(\pi(g) \pi(h) \xi) \quad \text { для любых } \quad g, h \in G, \quad \xi \in E, \quad f \in E^{*} .
$$

По непрерывности по переменной $g \in G$ и по определению продолжения мы получаем, что

$$
f(\pi(k h) \xi)=f(\pi(k) \pi(h) \xi) \quad \text { для любых } \quad k \in K, \quad h \in G, \quad \xi \in E, \quad f \in E^{*}
$$

или, что равносильно,

$$
\left(\pi(k h)^{*} f\right)(\xi)=\left(\pi(k)^{*} f\right)(\pi(h) \xi) \quad \text { для любых } k \in K, \quad h \in G, \quad \xi \in E, \quad f \in E^{*} .
$$

Так как непрерывность представления $\pi$ в пространстве $E$ в слабой топологии $\sigma\left(E, E^{*}\right)$ почти тавтологически равносильна непрерывности представления $g \mapsto \pi\left(g^{-1}\right)^{*}$ в пространстве $E^{*}$ в слабой топологии $\sigma\left(E^{*}, E\right)$ (с учетом непрерывности взятия обратного в группе $K)$, то

$$
\left(\pi(k l)^{*} f\right)(\xi)=\left(\pi(k)^{*} f\right)(\pi(l) \xi) \quad \text { для любых } \quad k, l \in K, \quad \xi \in E, \quad f \in E^{*}
$$

или, иначе говоря,

$$
f(\pi(k l) \xi)=f(\pi(k) \pi(l) \xi) \quad \text { для любых } \quad k, l \in K, \quad \xi \in E, \quad f \in E^{*} .
$$

Следовательно,

$$
\pi(k l)=\pi(k) \pi(l) \quad \text { для любых } \quad k, l \in K .
$$

Поскольку $\pi(e)=1_{E}$ по предположению, это завершает доказательство предложения 4 .

Использованное в доказательстве рассуждение можно почти дословно повторить для случая локально псевдокомпактных групп. Единственное отличие состоит в том, что нужно использовать псевдокомпактную окрестность любого элемента группы, а не группу в целом. Мы получаем следующее утверждение.

ПРЕДЛОЖЕНИЕ 5. Пусть топологическая группа $G$ локально псевдокомпактна, пусть $K$ - пополнение Вейля группь $G, E$ - квазиполное бочечное локально выпуклое пространство, а $\pi$ - слабо непрерывное представление группь $G$ в пространстве $E$. Тогда представление $\pi$ сильно непрерывно и представление $\pi$ можно продолжить до сильно непрерывного представления локально компактной группы $K$ в том же пространстве Е. Если исходная группа $G$ локально компактна, то утверждение остается справедливым, если условие квазиполноты пространства $E$ заменить условием, что пространство Е является пространством Крейна в слабой топологии.

Перейдем теперь к доказательству теоремы.

ДоКАЗАТЕЛЬСТВо ТЕОРЕМЫ 3 . Пусть $G$ - топологическая группа, имеющая замкнутую псевдокомпактную подгруппу $N$, для которой однородное пространство (пространство смежных классов) $G / N$ метризуемо. Пусть $K$ - пополнение Вейля группы $N$. Согласно предложению 4 ограничение представления $\pi$ на $N$ допускает слабо непрерывное продолжение на $K$, и это продолжение сильно 
непрерывно. Пусть $\xi \in E, f \in E^{*}, \operatorname{Re} f(\xi)>0$. Вследствие слабой непрерывности представления $\pi$ для любого $\varepsilon>0$ существует такая окрестность $V$ единицы в $K$, что

$$
\operatorname{Re} f(\pi(m) \xi)>\operatorname{Re} f(\xi)-\varepsilon \quad \text { для любых } m \in V,
$$

и такой компактный нормальный делитель $M$ в $K$, что $M \subset V$. Можно считать, что $0<\varepsilon<\operatorname{Re} f(\xi)$. Тогда из сильной непрерывности представления $\pi$ следует, что существует ненулевой $M$-инвариантный вектор

$$
\eta=\int_{M} \pi(m) \xi d \mu_{M}(m) \in E,
$$

который удовлетворяет условию $\operatorname{Re} f(\eta)>\operatorname{Re} f(\xi)-\varepsilon$. Это наблюдение показывает, что векторное подпространство $L$ пространства $E$, образованное неподвижными векторами $\zeta \in E$ всевозможных компактных $G_{\delta}$-подгрупп в $K$, слабо плотно в $E$, и поэтому $L$ сильно плотно в $E$ (поскольку $L-$ векторное подпространство, слабо плотное в $E$ ). Поэтому достаточно доказать, что каждый вектор $\zeta \in L$ сильно непрерывен (т.е. отображение

$$
g \mapsto \pi(g) \zeta, \quad g \in G,
$$

сильно непрерывно на $G)$. Действительно, пусть $\xi \in E, O$ - такая окрестность единицы в $G$, что множество $\pi(O)$ эквинепрерывно, и пусть $U-$ (сильная) окрестность нуля в $E$. Пусть $V$ - такая (сильная) окрестность нуля в $E$, что $V+V+V \subset U$. Используя эквинепрерывность множества $\pi(O)$, мы можем найти такую симметричную (сильную) окрестность $W$ нуля в $E$, что

$$
\pi(g) W \subset V \quad \text { для любых } g \in O .
$$

Пусть $\zeta$ - такой вектор в $E$, что $\zeta$ инвариантно относительно нормального делителя $M$ в $H$ и при этом $\zeta-\xi \in W$. Тогда

$\pi(g) \xi-\xi=\pi(g) \xi-\pi(g) \zeta+\pi(g) \zeta-\zeta+\zeta-\xi \in \pi(g) \zeta-\zeta+V+V \subset V+V+V \subset U$ для любых $g \in \Omega$, где $\Omega$ - такая окрестность единицы в $G$, что

$$
\pi(g) \zeta-\zeta \in V \quad \text { для любых } g \in \Omega .
$$

Такая окрестность $\Omega$ действительно существует, потому что $\zeta$ - сильно непрерывный вектор по построению.

Итак, рассмотрим отображение $G \rightarrow E$, определяемое формулой $g \mapsto \pi(g) \zeta$. Пусть $M$ - такой замкнутый нормальный делитель типа $G_{\delta}$ в $N$, что

$$
\pi(m) \zeta=\zeta \quad \text { для любых } m \in M .
$$

Согласно работе [10] факторгруппа $N / M$ компактна и удовлетворяет первой аксиоме счетности. Поэтому $N / M$ - компактное метризуемое пространство, и, так как пространство $G / N$ тоже метризуемо (и поэтому является $k$-пространством), из теоремы 3.7.18 в [16] следует, что естественное отображение факторизации $G / M \rightarrow G / N$, определенное формулой $g M \mapsto g N$ для любого $g \in G$, является совершенным отображением на метризуемое пространство (ср. теоремы 5.18 и 5.38 (е) в [36]), причем прообразы всех точек являются метризуемыми компактными множествами. По теореме 5.5.7 в [16] отсюда следует, что пространство смежных классов $G / M$ метризуемо. 
Если $\zeta$ не является сильно непрерывным вектором, то отображение $G / M \rightarrow E$, определенное формулой $g M \mapsto \pi(g) \zeta, g \in G$, разрывно. Если $g_{0} M$ - точка разрыва, то, поскольку пространство $G / M$ метрическое и, в частности, удовлетворяет первой аксиоме счетности, существует такая последовательность $g_{m} M \rightarrow g_{0} M, g_{m} \in G$, что $\pi\left(g_{m}\right) \zeta$ не стремится к $\pi\left(g_{0}\right) \zeta$. Заменяя $\zeta$ на $\pi\left(g_{0}\right) \zeta$, $M$ на $M^{\prime}=g_{0} M g_{0}^{-1}$ и $G / M$ на $G / M^{\prime}$, видим, что при такой замене мы можем заменить точку $g_{0}$ точкой $e_{G / M^{\prime}}$. С другой стороны, если $e=e_{G / M^{\prime}}$ - точка разрыва, то очевидно, что любая точка $g \in G / M^{\prime}$ тоже является точкой разрыва. Чтобы упростить обозначения, будем считать, что $e$ - точка разрыва отображения $G / M \rightarrow E$ для исходной группы $M$.

Рассмотрим замкнутую подгруппу $Q$ в $G$, порожденную элементами $g_{m}$, $m \in \mathbb{N}$. Пусть $P=Q \cap M$. Тогда $P$ - замкнутая подгруппа в $Q$ и естественное отображение однородных пространств $Q / P \rightarrow G / M$, определенное формулой $q P \mapsto q M$, взаимно однозначно. Образ этого отображения - подпространство метризуемого пространства. Следовательно, $Q / P$ сепарабельна и метризуема. По предположению рассматриваемое представление не является сильно непрерывным; следовательно, существует такая полунорма $p$ на $E$, что числовая последовательность $p\left(\pi\left(g_{m}\right) \zeta-\zeta\right), m \in \mathbb{N}$, не стремится к нулю. Но в этой ситуации можно применить построение, предложенное Брауном в [1; замечание 12], и заменить исходное представление слабо непрерывным не сильно непрерывным представлением в локально выпуклом пространстве, топология которого порождена счетным семейством полунорм (в частности, это пространство метризуемо). Кроме того, можно ограничиться метризуемым пространством, порожденным $Q / P$-орбитой вектора $\zeta$. Поскольку эта орбита является сепарабельным метризуемым пространством, мы можем предполагать, что рассматриваемое метризуемое пространство представления сепарабельно.

Докажем теперь, что постановку задачи можно упростить и свести рассмотрение к случаю, когда не только пространство представления, но и рассматриваемая группа метризуема и сепарабельна.

Рассмотрим псевдокомпактную подгруппу $M$. Как мы видели выше, пространство смежных классов $N / M$ - (несвязная, но конечнолистная) группа Ли и поэтому $M$ - замкнутое подмножество типа $G_{\delta}$ в $G$. Любая группа вида

$$
g_{i_{1}}^{-1} g_{i_{2}} \cdots g_{i_{2 n-1}}^{-1} g_{i_{2 n}} M g_{i_{2 n}}^{-1} g_{i_{2 n-1}} \cdots g_{i_{2}}^{-1} g_{i_{1}}
$$

(получаемая из $M$ сопряжением с элементом подмножества, плотного в $Q$ ) тоже является псевдокомпактным замкнутым подмножеством типа $G_{\delta}$ в $G$. Пересечение $R$ всех таких подгрупп очевидным образом тоже является замкнутым подмножеством типа $G_{\delta}$ в $G$. Кроме того, множество $R$ инвариантно относительно группы, порожденной элементами $g_{1}, \ldots, g_{n}$, и, следовательно, относительно замыкания объединения таких групп. Отсюда следует, что $P$ является подмножеством $R$. Действительно, $P$ инвариантно относительно автоморфизмов, определенных элементами самого множества $P$, и поэтому

$$
P \subset M \subset N \quad \Longrightarrow \quad g_{i_{1}}^{-1} g_{i_{2}} \cdots g_{i_{2 n-1}}^{-1} g_{i_{2 n}} M g_{i_{2 n}}^{-1} g_{i_{2 n-1}} \cdots g_{i_{2}}^{-1} g_{i_{1}} \in M \subset N .
$$

Таким образом, $R$ - замкнутый нормальный делитель в $N$, являющийся замкнутым $G_{\delta}$-множеством в $N$. Из [10; теорема 2.11$]$ следует, что $R$ - псевдокомпактная группа. Тогда из [10; замечание 2.7] вытекает, что факторгруппа 
$N / R$ компактна. Так как $R-G_{\delta}$-множество в $G$, то $N / R$ - сепарабельная (удовлетворяющая второй аксиоме счетности) компактная метризуемая группа. Заметим, что подгруппа $R$ инвариантна относительно всех автоморфизмов группы $N$, порожденных элементами подгруппы $Q$. Следовательно, семейство $Q R$ элементов вида $q r, q \in Q, r \in R$, является подгруппой, инвариантной относительно всех автоморфизмов, определяемых элементами подгрупп $Q$ и $R$. Рассмотрим группу $H=Q R$. По построению эта группа содержит семейство элементов $g_{m}, m \in \mathbb{N}$. Рассмотрим тогда семейство таких элементов $h \in H$, что $\pi(h) \zeta \equiv \zeta$. Переходя к подпространству векторов в $E$, которые остаются на месте под действием всех элементов нормального делителя $R$ (напомним, что мы уже знаем, что структура слабо непрерывных представлений группы $R$ совпадает со структурой слабо непрерывных представлений ее компактного пополнения Вейля), мы получаем слабо непрерывное представление факторгруппы $H / R$ в метризуемом пространстве $R$-инвариантных векторов. Это представление слабо непрерывно, но не сильно непрерывно.

Заметим, что в приведенной выше постановке группа $H / R$ метризуема и сепарабельна и пространство представления тоже метризуемо (т.е. пространство, порожденное орбитой вектора $\zeta$ ) и тоже сепарабельно (поскольку оно слабо сепарабельно по построению). Наконец, по построению полученное представление слабо непрерывно, но не сильно непрерывно. Но существование такого представления противоречит утверждению, полученному в первой части доказательства леммы 10 статьи Брауна [1; ч. 1] (т.е. противоречит "сепарабельной" части утверждения этой леммы). Это завершает доказательство теоремы 3.

Заметим, что аргументация Брауна, на которую мы неоднократно ссылались в доказательстве, основана на борелевских соображениях (ср. [39]-[42]), а не на свойствах измеримости. Кроме того, сильно измеримые функции в локально выпуклых пространствах общего вида образуют развитую иерархию (см., например, [43]), работа с которой требует значительных предварительных усилий.

В заключение параграфа приведем одно из простейших и важнейших следствий теоремы 3, которое непосредственно вытекает из этой теоремы.

СЛЕДСТвИЕ 3. Пусть $G$ - локально псевдокомпактная топологическая группа, $E$ - квазиполное бочечное локально выпуклое пространство, $\mathscr{L}(E)$ алгебра всех непрерывных линейных операторов в $E$, a $\pi$ - слабо непрерывное представление $G$ в пространстве $E$, причем $\pi$ локально слабо ограничено (т.е. образ $\pi(U)$ некоторой окрестности $U$ единицы в $G$ является слабо ограниченным множеством в $\mathscr{L}(E))$. Тогда $\pi$ - сильно непрерывное представление $G$ в $E$.

\section{§ 8. Заключительные замечания}

Отметим, что если представление некомпактной группы в “хорошем" топологическом векторном пространстве (и даже в гильбертовом пространстве) имеет такое инвариантное подпространство, что представления в этом подпространстве и в соответствующем факторпространстве, скажем, сильно непрерывны, то все представление не обязательно слабо или сильно непрерывно. Соот- 
ветствующий пример, приведенный Брауном [1], использует двумерное треугольное представление аддитивной группы вещественных чисел с единицами на диагонали и разрывным аддитивным характером в наддиагональной клетке. Поэтому “условие (5)" статьи [1] или его аналог - локальная эквинепрерывность - являются существенными условиями слабой непрерывности.

Отметим также, что мы считаем слабо непрерывное представление заданным и не обсуждаем здесь условий существования таких представлений (так называемой представимости, т.е. существования представлений с теми или иными алгебраическими или топологическими свойствами). Существен результат Телемана [44], утверждающий, что любая хаусдорфова топологическая группа имеет точное слабо непрерывное представление в банаховом пространстве. Известен и следующий критерий "рефлексивной представимости” (полученный с помощью результатов статей [45] и [46]), похожий по форме на критерий конечномерной представимости топологической группы в терминах ее непрерывного вложения в компактную группу: хаусдорфова топологическая группа имеет достаточно много непрерывных представлений (т.е. семейство представлений, разделяющее элементы группы) в рефлексивных банаховых пространствах тогда и только тогда, когда она допускает непрерывное вложение в компактную полутопологическую полугруппу [47]. Но проблема "унитарной представимости" безусловно нетривиальна за пределами применимости теоремы Гельфанда-Райкова, и необходимые и достаточные условия существования достаточно большого семейства непрерывных представлений в гилъбертовых пространствах еще неизвестны (в связи с этим есть интересный отрицательный результат: группа, вложимая в компактную полутопологическую полугруппу, может не быть унитарно представимой [48], и имеется ряд примеров групп, не имеющих никаких непрерывных унитарных представлений в гильбертовых пространствах [49]). Описание топологических групп, имеющих достаточно много конечномерных унитарных представлений, которыми список неприводимых представлений в гильбертовом пространстве исчерпывается (т.е. все топологически неприводимые унитарные представления этих групп в гильбертовых пространствах конечномерны), приведено в работе [50].

Автор благодарен Лоуренсу Брауну за чрезвычайно полезный комментарий к его работе [1] и А. С. Мищенко за внимание к работе.

\section{Список литературы}

[1] L. G. Brown, "Continuity of actions of groups and semigroups on Banach spaces", J. London Math. Soc. (2), 62:1 (2000), 107-116.

[2] K. Thomsen, "Asymptotic homomorphisms and equivariant $K K$-theory", J. Funct. Anal., 163:2 (1999), 324-343.

[3] K. Thomsen, "Nonstable K-theory for operator algebras", K-Theory, 4:3 (1991), 245-267.

[4] J. Cuntz, "Bivariante $K$-Theorie für lokalkonvexe Algebren und der Chern-ConnesCharakter", Doc. Math., 2 (1997), 139-182.

[5] J. Cuntz, Bivariant K-theory and the Weyl algebra, arXiv: math.KT/0401295.

[6] E. J. Beggs, Pointwise bounded asymptotic morphisms and Thomsen's non-stable $k$ theory, arXiv: math.0A/0201051. 
[7] A. I. Shtern, "Representations of topological groups in locally convex spaces: continuity properties and weak almost periodicity", Russian J. Math. Phys., 11:1 (2004), 81-108.

[8] Х. Шефер, Топологические векторные пространства, Мир, М., 1971.

[9] W. W. Comfort, K. A. Ross, "Pseudocompactness and uniform continuity in topological groups", Pacific J. Math., 16:3 (1966), 483-496.

[10] W. W. Comfort, F. J. Trigos-Arrieta, "Locally pseudocompact topological groups", Topology Appl., 62:3 (1995), 263-280.

[11] D. Dikranjan, D. Shakhmatov, Algebraic structure of pseudocompact groups, Mem. Amer. Math. Soc., 633, Amer. Math. Soc., Providence, RI, 1998.

[12] S. A. Gaal, Linear analysis and representation theory, Springer-Verlag, New York, 1973.

[13] M. A. Naimark, A. I. Štern [Shtern], Theory of group representations, Springer-Verlag, New York-Heidelberg-Berlin, 1982.

[14] F.P. Greenleaf, Invariant means on topological groups and their applications, Van Nostrand Reinhold Co., London, 1969.

[15] A. T.-M. Lau, "Amenability of semigroups", The analytical and topological theory of semigroups, de Gruyter Exp. Math., 1, de Gruyter, Berlin, 1990, 313-334.

[16] Р. Энгелькинг, Общая топология, Мир, М., 1986.

[17] М. М. Дэй, Нормированнъе линейные пространства, Наука, М., 1961.

[18] C. C. Moore, "Extensions and low dimensional cohomology theory of locally compact groups. I, II", Trans. Amer. Math. Soc., 113 (1964), 40-63, 64-86.

[19] M. Krein, V. Śmulian, "On regularly convex sets in the space conjugate to a Banach space", Ann. of Math. (2), 41 (1940), 556-583.

[20] J. von Neumann, "On complete topological spaces", Trans. Amer. Math. Soc., 37 (1935), 1-20.

[21] K. de Leeuw, I. Glicksberg, "The decomposition of certain group representations", J. Anal. Math., 15 (1965), 135-192.

[22] B. E. Johnson, Cohomology of Banach algebras, Mem. Amer. Math. Soc., 127, Amer. Math. Soc., Providence, RI, 1972.

[23] Ю. И. Любич, Введение в теорию банаховых представлений групп, Вища школа, Харьков, 1985.

[24] M. G. Megrelishvili, "Operator topologies and reflexive representability", Nuclear groups and Lie groups, Selected lectures of the workshop (Madrid, Spain, September 1999), Res. Exp. Math., 24, eds. E. Martin Peinador, J. Nunez Garcia, Heldermann, Lemgo, 2001, 197-208.

[25] M. G. Megrelishvili, "Fragmentability and continuity of semigroup actions", Semigroup Forum, 57:1 (1998), 101-126.

[26] L. Egghe, "On the Radon-Nikodym property, and related topics in locally convex spaces", Lecture Notes in Phys., 77 (1978), 77-90.

[27] R. C. James, "KMP, RNP, and PCP for Banach spaces", Banach space theory (Iowa City, IA, 1987), Contemp. Math., 85, Amer. Math. Soc., Providence, RI, 1989, 281317.

[28] I. Namioka, "Radon-Nikodym compact spaces and fragmentability", Mathematika, 34 (1987), 258-281.

[29] А. И. Штерн, "Критерии слабой и сильной непрерывности представлений топологических групп в банаховых пространствах", Матем. сб., 193:9 (2002), 139-156.

[30] А.И. Штерн, “Жесткость и аппроксимация квазипредставлений аменабельных групп", Матем. заметки, 65:6 (1999), 908-920.

[31] S. Antonyan, M. Sanchis, "Extension of locally pseudocompact group actions", Ann. Mat. Pura Appl. (4), 181 (2002), 239-246. 
[32] A. V. Arhangel'skii, "Moscow spaces, Pestov-Tkačenko problem, and $C$-embeddings", Comment. Math. Univ. Carolin., 41:3 (2000), 585-595.

[33] M. Sanchis, "Continuous functions on locally pseudocompact groups", Topology Appl., 86:1 (1998), 5-23.

[34] L. G. Brown, "Topologically complete groups", Proc. Amer. Math. Soc., 35:2 (1972), 593-600.

[35] Б. А. Пасынков, "Почти метризуемые топологические группы", Докл. АН CCCP, 161:2 (1965), 281-284.

[36] Э. Хьюитт, К. А. Росс, Абстрактный гармонический анализ, т. I, Мир, М., 1975.

[37] M. Valdivia, "Some new results on weak compactness", J. Funct. Anal., 24:1 (1977), $1-10$.

[38] A. Grothendieck, "Critères de compacité dans les espaces fonctionnels généraux", Amer. J. Math., 74 (1952), 168-186.

[39] S. Banach, Théorie des opérations linéaires, Subwencji Funduszu Narodowej, Warszawa, 1932.

[40] K.-H. Neeb, "On a theorem of S. Banach", J. Lie Theory, 7:2 (1997), 293-300.

[41] K.-H. Neeb, D. Pickrell, "Supplements to the papers entitled: "On a theorem of S. Banach" and "The separable representations of $U(H)$ ", J. Lie Theory, 10:1 (2000), 107-109.

[42] V. Pestov, Review of [40], MR1473172.

[43] S. S. Khurana, "Pointwise compactness and measurability", Pacific J. Math., 83:2 (1979), 387-391.

[44] S. Teleman, "Sur la réprésentation linéaire des groupes topologiques", Ann. Sci. École Norm. Sup. (4), 74 (1957), 319-339.

[45] N. J. Young, "Periodicity of functionals and representations of normed algebras on reflexive spaces", Proc. Edinburgh Math. Soc. (2), 20:2 (1976/77), 99-120.

[46] W. J. Davis, T. Figiel, W. B. Johnson, A. Pelczynski, "Factoring weakly compact operators", J. Funct. Anal., 17 (1974), 311-327.

[47] A.I. Shtern, "Compact semitopological semigroups and reflexive representability of topological groups", Russian J. Math. Phys., 2:1 (1994), 131-132.

[48] M. Megrelishvili, "Generalized Heisenberg groups and Shtern's question", Georgian Math. J., 11:4 (2004), 775-782.

[49] W. Banaszczyk, Additive subgroups of topological vector spaces, Lecture Notes in Math., 1466, Springer-Verlag, Berlin, 1991.

[50] А. И. Штерн, “Топологические группы с конечными групповыми алгебрами фон Неймана типа I", Матем. сб., 196:3 (2005), 143-160.

А. И. Штерн (А. I. Shtern)

Московский государственный университет

Поступила в редакцию

им. М. В. Ломоносова

25.02 .2005

E-mail: ashtern@member.ams.org 